\title{
On Ulam's Type Stability of the Cauchy Additive Equation
}

\author{
Janusz Brzdęk \\ Department of Mathematics, Pedagogical University, Podchorążych 2, 30-084 Kraków, Poland \\ Correspondence should be addressed to Janusz Brzdęk; jbrzdek@up.krakow.pl
}

Received 27 August 2013; Accepted 8 December 2013; Published 16 March 2014

Academic Editors: N. Kallur and P. Yang

Copyright (c) 2014 Janusz Brzdęk. This is an open access article distributed under the Creative Commons Attribution License, which permits unrestricted use, distribution, and reproduction in any medium, provided the original work is properly cited.

We prove a general result on Ulam's type stability of the functional equation $f(x+y)=f(x)+f(y)$, in the class of functions mapping a commutative group into a commutative group. As a consequence, we deduce from it some hyperstability outcomes. Moreover, we also show how to use that result to improve some earlier stability estimations given by Isaac and Rassias.

\section{Introduction}

The issue of stability of functional equations has been a very popular subject of investigations for the last nearly fifty years (see, e.g., [1-8]). Its main motivation was given by Ulam (cf. [9-11]) in 1940 in his talk at the University of Wisconsin. For instance, we can introduce the following definition, which somehow describes the main ideas of such stability notion for equations in two variables $\left(\mathbb{R}_{+}\right.$stands for the set of nonnegative reals).

Definition 1. Let $A$ be a nonempty set, $(X, d)$ be a metric space, $\mathscr{C} \subset \mathbb{R}_{+}^{A^{2}}$ be nonempty, $\mathscr{T}$ be an operator mapping $\mathscr{C}$ into $\mathbb{R}_{+}^{A}$, and $\mathscr{F}_{1}, \mathscr{F}_{2}$ be operators mapping nonempty $\mathscr{D} \subset X^{A}$ into $X^{A^{2}}$. We say that the equation

$$
\mathscr{F}_{1} \varphi(x, y)=\mathscr{F}_{2} \varphi(x, y)
$$

is $\mathscr{T}$-stable provided for every $\varepsilon \in \mathscr{C}$ and $\varphi_{0} \in \mathscr{D}$ with

$$
d\left(\mathscr{F}_{1} \varphi_{0}(x, y), \mathscr{F}_{2} \varphi_{0}(x, y)\right) \leq \varepsilon(x, y) \quad x, y \in A,
$$

there exists a solution $\varphi \in \mathscr{D}$ of (1) such that

$$
d\left(\varphi(x), \varphi_{0}(x)\right) \leq \mathscr{T} \varepsilon(x) \quad x \in A .
$$

(As usual, $C^{D}$ denotes the family of all functions mapping a set $D \neq \emptyset$ into a set $C \neq \emptyset$.) Roughly speaking, $\mathscr{T}$-stability of
(1) means that every approximate (in the sense of (2)) solution to (1) is always close (in the sense of (3)) to an exact solution to (1). The next theorem is an example of the most classical results.

Theorem 2. Let $E_{1}$ and $E_{2}$ be two normed spaces and let $c \geq$ 0 and $p \neq 1$ be fixed real numbers. Let $f: E_{1} \rightarrow E_{2}$ be an operator such that

$$
\begin{aligned}
& \|f(x+y)-f(x)-f(y)\| \\
& \quad \leq c\left(\|x\|^{p}+\|y\|^{p}\right) \quad x, y \in E_{1} \backslash\{0\} .
\end{aligned}
$$

If $p \geq 0$ and $E_{2}$ is complete, then there is a unique operator $T: E_{1} \rightarrow E_{2}$ that is additive (i.e., $T(x+y)=T(x)+T(y)$ for $\left.x, y \in E_{1}\right)$ and such that

$$
\|f(x)-T(x)\| \leq \frac{c\|x\|^{p}}{\left|2^{p-1}-1\right|} \quad x \in E_{1} \backslash\{0\} .
$$

If $p<0$, then $f$ is additive.

It has been motivated by Rassias (see [12-14]) and is composed of the outcomes in [15-17]. Note that Theorem 2 with $p=0$ yields the result of Hyers [9] and it is known (see [17]; cf. also [18, 19]) that for $p=1$ an analogous result is not valid. Moreover, it has been shown in [20] that estimation (5) is optimum for $p \geq 0$ in the general case. 
The second statement of Theorem 2, for $p<0$, can be described as the $\varphi$-hyperstability of the additive Cauchy equation for $\varphi(x, y) \equiv c\left(\|x\|^{p}+\|y\|^{p}\right.$ ) (for further information on hyperstability see, e.g., $[1,16,21,22]$; some other recent results can be found in [23-25]). It seems to be of interest that such result does not remain valid if we restrict the domain of $f$ to a subsemigroup of the group $\left(E_{1},+\right)$. The subsequent remark shows this.

Remark 3. Let $p<0, a \geq 0, I=(a, \infty)$, and $f, T: I \rightarrow \mathbb{R}$ be given by $T(x)=0$ and $f(x)=x^{p}$ for $x \in I$. Then clearly

$$
|f(x)-T(x)|=x^{p} \quad x \in I .
$$

Moreover,

$$
|f(x+y)-f(x)-f(y)| \leq x^{p}+y^{p} \quad x, y \in I .
$$

In fact, suppose, for instance, that $x \leq y$. Then $(x+y)^{p} \leq$ $(2 x)^{p}=2^{p} x^{p} \leq x^{p} \leq x^{p}+y^{p}$, whence $\mid f(x+y)-f(x)-$ $f(y) \mid=x^{p}+y^{p}-(x+y)^{p} \leq x^{p}+y^{p}$.

In this paper we prove a quite general result that allows us to generalize and extend Theorem 2 in various directions.

\section{An Auxiliary Result}

In the proof of the main theorem in this paper, we use the following fixed point result that can be easily derived from [26, Theorem 2] (cf. [27, Theorem 1] and [28]). For a survey on applications of the fixed point methods for similar issues, see [29].

Theorem 4. Assume that $Z$ is a nonempty set, $(Y, d)$ is a complete metric space, $f_{1}, f_{2}: Z \rightarrow Z, \mathscr{T}: Y^{Z} \rightarrow Y^{Z}$ is an operator satisfying the inequality

$$
\begin{array}{r}
d(\mathscr{T} \xi(x), \mathscr{T} \mu(x)) \leq d\left(\xi\left(f_{1}(x)\right), \mu\left(f_{1}(x)\right)\right) \\
+d\left(\xi\left(f_{2}(x)\right), \mu\left(f_{2}(x)\right)\right), \\
\xi, \mu \in Y^{Z}, x \in Z,
\end{array}
$$

and $\Lambda: \mathbb{R}_{+}^{Z} \rightarrow \mathbb{R}_{+}^{Z}$ is an operator defined by

$$
\Lambda \delta(x):=\delta\left(f_{1}(x)\right)+\delta\left(f_{2}(x)\right) \quad \delta \in \mathbb{R}_{+}^{Z}, x \in Z .
$$

Suppose that there exist functions $\varepsilon: Z \rightarrow \mathbb{R}_{+}$and $\varphi: Z \rightarrow$ $Y$ such that

$$
\begin{gathered}
d(\mathscr{T} \varphi(x), \varphi(x)) \leq \varepsilon(x), \\
\varepsilon^{*}(x):=\sum_{n=0}^{\infty}\left(\Lambda^{n} \varepsilon\right)(x)<\infty \quad x \in Z,
\end{gathered}
$$

where $\Lambda^{n}$ denotes the $n$th iterate of $\Lambda$ (i.e., $\Lambda^{0} \delta=\delta$ for $\delta \in \mathbb{R}_{+}{ }^{Z}$ and $\Lambda^{n}:=\Lambda \bigcirc \Lambda^{n-1}$ for $n \in \mathbb{N}$ ). Then there exists a unique fixed point $\psi$ of $\mathscr{T}$ with

$$
d(\varphi(x), \psi(x)) \leq \varepsilon^{*}(x) \quad x \in Z .
$$

Moreover,

$$
\psi(x):=\lim _{n \rightarrow \infty}\left(\mathscr{T}^{n} \varphi\right)(x) \quad x \in Z
$$

\section{The Main Theorem}

Given a group $(X,+)$, we denote by $A$ ut $X$ the family of all automorphisms of $X$. Moreover, for each $u \in X^{X}$ we write $u x:=u(x)$ for $x \in X$ and we define $u^{\prime} \in X^{X}$ by $u^{\prime} x:=x-u x$.

The next theorem is the main result of this paper.

Theorem 5. Let $(X,+)$ and $(E,+)$ be commutative groups, $d$ be a complete metric in $E$ that is invariant (i.e., $d(x+z, y+z)=$ $d(x, y)$ for $x, y, z \in X), H:(X \backslash\{0\})^{2} \rightarrow \mathbb{R}_{+}$, and

$l(X)$

$$
:=\left\{u \in \text { Aut } X: u^{\prime} \in \text { Aut } X, \lambda\left(u^{\prime}\right)+\lambda(u)<1\right\} \neq \emptyset,
$$

where

$$
\begin{aligned}
\lambda(u):=\inf \{t & \in \mathbb{R}_{+}: H(u x, u y) \\
& \leq t H(x, y) \forall x, y \in X \backslash\{0\}\}
\end{aligned}
$$

for $u \in$ Aut $X$. Assume that $f: X \rightarrow$ Esatisfies the inequality

$$
d(f(x+y), f(x)+f(y)) \leq H(x, y) \quad x, y \in X \backslash\{0\} .
$$

Then, for each nonempty $\mathcal{U} \subset l(X)$ such that

$$
u \bigcirc v=v \bigcirc u \quad u, v \in \mathcal{U}
$$

there exists a unique additive $T: X \rightarrow E$ fulfilling the inequality

$$
d(f(x), T(x)) \leq H_{\mathscr{U}}(x) \quad x \in X \backslash\{0\},
$$

where

$$
H_{\mathcal{U}}(x):=\inf \left\{\frac{H\left(u^{\prime} x, u x\right)}{1-\lambda(u)-\lambda\left(u^{\prime}\right)}: u \in \mathcal{U}\right\} \quad x \in X \backslash\{0\}
$$

Proof. Let $\mathcal{U} \subset l(X)$ be nonempty and let (16) be valid. Write $X_{0}:=X \backslash\{0\}$. Note that (15), with $x$ replaced by $u^{\prime} x$ and $y=u x$, gives

$$
\begin{aligned}
& d\left(f(x), f\left(u^{\prime} x\right)+f(u x)\right) \\
& \quad \leq H\left(u^{\prime} x, u x\right) \quad x \in X_{0}, u \in \mathcal{U} .
\end{aligned}
$$

Given $u \in \mathcal{U}$, we define operators $\mathscr{T}_{u}: E^{X_{0}} \rightarrow E^{X_{0}}$ and $\Lambda_{u}: \mathbb{R}_{+}^{X_{0}} \rightarrow \mathbb{R}_{+}^{X_{0}}$ by

$$
\mathscr{T}_{u} \xi(x):=\xi\left(u^{\prime} x\right)+\xi(u x) \quad x \in X_{0}, \xi \in E^{X_{0}}, u \in \mathcal{U},
$$

$$
\Lambda_{u} \delta(x):=\delta\left(u^{\prime} x\right)+\delta(u x) \quad x \in X_{0}, \delta \in \mathbb{R}_{+}^{X_{0}}, u \in \mathcal{U}
$$


It is easily seen that, for each $u \in \mathcal{U}, \Lambda:=\Lambda_{u}$ has form (9) with $Z:=X_{0}, f_{1}(x)=u^{\prime} x$, and $f_{2}(x)=u x$. Moreover, (19) can be written in the following way

$$
\begin{aligned}
d\left(f(x), \mathscr{T}_{u} f(x)\right) & \leq H\left(u^{\prime} x, u x\right) \\
& =: \varepsilon_{u}(x) \quad x \in X_{0}, u \in \mathcal{U} .
\end{aligned}
$$

(Here and in the sequel, the restriction of $f$ to the set $X_{0}$ is also denoted by $f$; we believe that this will not cause any confusion.) And

$$
\begin{aligned}
d\left(\mathscr{T}_{u} \xi(x), \mathscr{T}_{u} \mu(x)\right) \\
\quad=d\left(\xi\left(u^{\prime} x\right)+\xi(u x), \mu\left(u^{\prime} x\right)+\mu(u x)\right) \\
\quad \leq d\left(\xi\left(u^{\prime} x\right), \mu\left(u^{\prime} x\right)\right)+d(\xi(u x), \mu(u x))
\end{aligned}
$$

for every $\xi, \mu \in E^{X_{0}}, x \in X_{0}$, and $u \in \mathcal{U}$. Consequently, for each $u \in \mathcal{U}$, also (8) is valid with $Z:=X_{0}, Y:=E$, and $\mathscr{T}:=\mathscr{T}_{u}$.

Note that, in view of the definition of $\lambda(u)$,

$$
H(u x, u y) \leq \lambda(u) H(x, y) \quad u \in \mathcal{U}, x, y \in X_{0} .
$$

So, it is easy to show by induction on $n$ that

$$
\Lambda_{u}^{n} \varepsilon_{u}(x) \leq\left(\lambda\left(u^{\prime}\right)+\lambda(u)\right)^{n} H\left(u^{\prime} x, u x\right)
$$

for $x \in X_{0}, n \in \mathbb{N}_{0}$ (nonnegative integers), and $u \in \mathcal{U}$. Hence,

$$
\begin{aligned}
\varepsilon_{u}^{*}(x): & =\sum_{n=0}^{\infty}\left(\Lambda_{u}^{n} \varepsilon_{u}\right)(x) \\
& \leq H\left(u^{\prime} x, u x\right) \sum_{n=0}^{\infty}\left(\lambda\left(u^{\prime}\right)+\lambda(u)\right)^{n} \\
& =\frac{H\left(u^{\prime} x, u x\right)}{1-\lambda(u)-\lambda\left(u^{\prime}\right)} \quad x \in X_{0}, u \in \mathcal{U} .
\end{aligned}
$$

Now, we can use Theorem 4 with $Z=X_{0}, Y=E, \varepsilon:=\varepsilon_{u}$, and $\varphi=f$. According to it, the limit

$$
T_{u}^{\prime}(x):=\lim _{n \rightarrow \infty}\left(\mathscr{T}_{u}^{n} f\right)(x)
$$

exists for each $x \in X_{0}$ and $u \in \mathcal{U}$,

$$
d\left(f(x), T_{u}(x)\right) \leq \frac{H\left(u^{\prime} x, u x\right)}{1-\lambda(u)-\lambda\left(u^{\prime}\right)} \quad x \in X_{0}, u \in \mathscr{U},
$$

and the function $T_{u}: X \rightarrow E$ defined by

$$
T_{u}(0)=0, \quad T_{u}(x):=T_{u}^{\prime}(x) \quad x \in X_{0}
$$

is a solution of the equation

$$
T(x)=T\left(u^{\prime} x\right)+T(u x),
$$

because $T_{u}^{\prime}$ is a fixed point of $\mathscr{T}_{u}$.
Now we show that

$$
\begin{gathered}
d\left(\mathscr{T}_{u}^{n} f(x+y), \mathscr{T}_{u}^{n} f(x)+\mathscr{T}_{u}^{n} f(y)\right) \\
\leq\left(\lambda\left(u^{\prime}\right)+\lambda(u)\right)^{n} H(x, y)
\end{gathered}
$$

for every $x, y \in X_{0}, x+y \neq 0, n \in \mathbb{N}_{0}$, and $u \in \mathcal{U}$.

Since the case $n=0$ is just (15), take $k \in \mathbb{N}_{0}$ and assume that (31) holds for $n=k$ and every $x, y \in X_{0}, x+y \neq 0$, and $u \in \mathcal{U}$. Then, by (24),

$$
\begin{array}{rl}
d\left(\mathscr{T}_{u}^{k+1} f(x+y), \mathscr{T}_{u}^{k+1} f(x)+\mathscr{T}_{u}^{k+1} f(y)\right) & d\left(\mathscr{T}_{u}^{k} f\left(u^{\prime}(x+y)\right)+\mathscr{T}_{u}^{k} f(u(x+y)),\right. \\
& \mathscr{T}_{u}^{k} f\left(u^{\prime} x\right)+\mathscr{T}_{u}^{k} f(u x)+\mathscr{T}_{u}^{k} f\left(u^{\prime} y\right) \\
& \left.+\mathscr{T}_{u}^{k} f(u y)\right) \\
\leq & d\left(\mathscr{T}_{u}^{k} f\left(u^{\prime} x+u^{\prime} y\right), \mathscr{T}_{u}^{k} f\left(u^{\prime} x\right)+\mathscr{T}_{u}^{k} f\left(u^{\prime} y\right)\right) \\
& +d\left(\mathscr{T}_{u}^{k} f(u x+u y), \mathscr{T}_{u}^{k} f(u x)+\mathscr{T}_{u}^{k} f(u y)\right) \\
\leq & \left(\lambda\left(u^{\prime}\right)+\lambda(u)\right)^{k} H\left(u^{\prime} x, u^{\prime} y\right) \\
& +\left(\lambda\left(u^{\prime}\right)+\lambda(u)\right)^{k} H(u x, u y) \\
\leq & \left(\lambda\left(u^{\prime}\right)+\lambda(u)\right)^{k+1} H(x, y) \\
x & x, y \in X_{0}, \quad x+y \neq 0, \quad u \in \mathscr{U} .
\end{array}
$$

Thus, by induction, we have shown that (31) holds for every $x, y \in X_{0}, x+y \neq 0, n \in \mathbb{N}_{0}$, and $u \in \mathcal{U}$. Letting $n \rightarrow \infty$ in (31), we obtain the equality

$$
\begin{aligned}
& T_{u}(x+y)=T_{u}(x)+T_{u}(y) \\
& x, y \in X_{0}, \quad x+y \neq 0, \quad u \in \mathcal{U} .
\end{aligned}
$$

From this we can deduce that $T_{u}$ is additive for each $u \in$ $\mathcal{U}$. The reasoning is very simple, but for the convenience of readers we present it here.

In view of (33), it is only enough to consider the situation $y=-x$. So take $u \in \mathcal{U}$ and $x \in X_{0}$ (the case $x=0$ is trivial). Then, by (33),

$$
\begin{aligned}
T_{u}(x) & =T_{u}(x+x-x) \\
& =T_{u}(2 x)+T_{u}(-x) \\
& =2 T_{u}(x)+T_{u}(-x),
\end{aligned}
$$

which yields $T_{u}(x)+T_{u}(-x)=0$ and consequently $T_{u}(x-x)=$ $T_{u}(0)=0=T_{u}(x)+T_{u}(-x)$.

Next, we prove that each additive $T: X \rightarrow Y$ satisfying the inequality

$$
d(f(x), T(x)) \leq L H\left(v x, v^{\prime} x\right) \quad x \in X_{0},
$$


with some $L>0$ and $v \in \mathcal{U}$, is equal to $T_{w}$ for each $w \in \mathcal{U}$. To this end fix $v, w \in \mathcal{U}, L>0$, and an additive $T: X \rightarrow Y$ satisfying (35). Note that, by (28) and (35), there is $L_{0}>0$ such that

$$
\begin{aligned}
d\left(T(x), T_{w}(x)\right) \leq & d(T(x), f(x))+d\left(f(x), T_{w}(x)\right) \\
\leq & L_{0}\left(H\left(v^{\prime} x, v x\right)+H\left(w^{\prime} x, w x\right)\right) \\
& \times \sum_{n=0}^{\infty}\left(\lambda\left(w^{\prime}\right)+\lambda(w)\right)^{n}
\end{aligned}
$$

for $x \in X_{0}$. Observe yet that $T$ and $T_{w}$ are solutions to (30) for all $u \in \mathcal{U}$, because they are additive.

We show that, for each $j \in \mathbb{N}_{0}$,

$$
\begin{aligned}
d\left(T(x), T_{w}(x)\right) & \\
\leq & L_{0}\left(H\left(v^{\prime} x, v x\right)+H\left(w^{\prime} x, w x\right)\right) \\
& \times \sum_{n=j}^{\infty}\left(\lambda\left(w^{\prime}\right)+\lambda(w)\right)^{n} \quad x \in X_{0} .
\end{aligned}
$$

The case $j=0$ is exactly (36). So fix $l \in \mathbb{N}_{0}$ and assume that (37) holds for $j=l$. Then, in view of (24),

$$
\begin{aligned}
d\left(T(x), T_{w}(x)\right) & d\left(T(w x)+T\left(w^{\prime} x\right), T_{w}(w x)+T_{w}\left(w^{\prime} x\right)\right) \\
\leq & d\left(T(w x), T_{w}(w x)\right)+d\left(T\left(w^{\prime} x\right), T_{w}\left(w^{\prime} x\right)\right) \\
\leq & L_{0}\left(H\left(v^{\prime} w x, v w x\right)+H\left(w^{\prime} w x, w w x\right)\right) \\
& \times \sum_{n=j}^{\infty}\left(\lambda\left(w^{\prime}\right)+\lambda(w)\right)^{n} \\
& +L_{0}\left(H\left(v^{\prime} w^{\prime} x, v w^{\prime} x\right)+H\left(w^{\prime} w^{\prime} x, w w^{\prime} x\right)\right) \\
& \times \sum_{n=j}^{\infty}\left(\lambda\left(w^{\prime}\right)+\lambda(w)\right)^{n} \\
\leq & L_{0}\left(H\left(v^{\prime} x, v x\right)+H\left(w^{\prime} x, w x\right)\right)\left(\lambda(w)+\lambda\left(w^{\prime}\right)\right) \\
& \times \sum_{n=j}^{\infty}\left(\lambda\left(w^{\prime}\right)+\lambda(w)\right)^{n} \\
= & L_{0}\left(H\left(v^{\prime} x, v x\right)+H\left(w^{\prime} x, w x\right)\right) \\
& \times \sum_{n=l+1}^{\infty}\left(\lambda\left(w^{\prime}\right)+\lambda(w)\right)^{n} x \in X_{0} .
\end{aligned}
$$

Thus we have shown (37). Now, letting $j \rightarrow \infty$ in (37), we get

$$
T(x)=T_{w}(x) \quad x \in X_{0} .
$$

Since $T$ and $T_{w}$ are additive, we have $T=T_{w}$.
In this way, we also have proved that $T_{u}=T_{w}$ for each $u \in \mathcal{U}$ (on account of (28)), which yields

$$
d\left(f(x), T_{w}(x)\right) \leq \frac{H\left(u^{\prime} x, u x\right)}{1-\lambda\left(u^{\prime}\right)-\lambda(u)} \quad x \in X_{0}, u \in \mathscr{U}
$$

This implies (17) with $T:=T_{w}$; clearly, equality (39) means the uniqueness of $T$, as well.

Thus we have completed the proof of Theorem 5 .

\section{Some Consequences}

Theorem 5 yields the subsequent corollary.

Corollary 6. Let $X, H, E$, and $d$ be as in Theorem 5. Suppose that there exists a nonempty $\mathcal{U} \subset l(X)$ such that (16) holds and

$$
\begin{gathered}
\inf _{u \in \mathscr{U}} H\left(u^{\prime} x, u x\right)=0 \quad x \in X \backslash\{0\}, \\
\sup _{u \in \mathscr{U}} \lambda\left(u^{\prime}\right)+\lambda(u)<1 .
\end{gathered}
$$

Then every $f: X \rightarrow$ E satisfying (15) is additive.

Proof. Suppose that $f: X \rightarrow E$ satisfies (15). Then, by Theorem 5, there exists an additive $T: X \rightarrow E$ such that (17) holds. Since, in view of (41), $H_{\mathcal{U}}(x)=0$ for $x \in X \backslash\{0\}$, this means that $f(x)=T(x)$ for $x \in X \backslash\{0\}$, whence

$$
f(x+y)=f(x)+f(y) \quad x, y \in X \backslash\{0\}, x+y \neq 0,
$$

which implies that $f$ is additive (see the proof of (34)).

The next corollary corresponds to the results on the inhomogeneous Cauchy equation (44) in [30-35].

Corollary 7. Let $X, H, E$, and $d$ be as in Theorem 5 and $F$ : $X^{2} \rightarrow$ E. Suppose that

$$
d(F(x, y), 0) \leq H(x, y) \quad x, y \in X,
$$

$F\left(x_{0}, y_{0}\right) \neq 0$ for some $x_{0}, y_{0} \in X \backslash\{0\}$, and there exists $a$ nonempty $\mathcal{U} \subset l(X)$ such that (16) and (41) hold. Then the inhomogeneous Cauchy equation

$$
f(x+y)=f(x)+f(y)+F(x, y)
$$

has no solutions in the class of functions $f: X \rightarrow E$.

Proof. Suppose that $f: X \rightarrow E$ is a solution to (44). Then

$$
\begin{aligned}
d(f(x+y), f(x)+f(y)) & \\
\quad= & d(f(x)+f(y)+F(x, y), f(x)+f(y)) \\
\quad & =d(F(x, y), 0) \leq H(x, y) \quad x, y \in X \backslash\{0\} .
\end{aligned}
$$

Consequently, by Corollary 6, $f$ is additive, whence $F\left(x_{0}, y_{0}\right)=f\left(x_{0}+y_{0}\right)-f\left(x_{0}\right)-f\left(y_{0}\right)=0$, which is a contradiction. 
Remark 8. We have excluded $x=0$ and $y=0$ from the domain of $H$, in Theorem 5, because of the reason which can be easily deduced from the subsequent natural example.

In the rest of this paper, we assume that $E_{1}$ and $E$ are normed spaces, $X$ is a subgroup of the group $\left(E_{1},+\right)$, with $d(x, y)=\|x-y\|$, and $H:(X \backslash\{0\})^{2} \rightarrow \mathbb{R}_{+}$. For each $n \in \mathbb{Z}$ define $\mu_{n}: X \rightarrow X$ by $\mu_{n} x:=n x$ for $x \in X$. Let $H$ be given by

$$
H(x, y)=c\|x\|^{p}+d\|y\|^{q} \quad x, y \in X \backslash\{0\}
$$

with some real $p<0, q<0, c \geq 0$, and $d \geq 0$. Then

$$
\begin{aligned}
H\left(\mu_{n} x, \mu_{k} y\right) & =H(n x, k y) \\
& =c\|n x\|^{p}+d\|k y\|^{q} \\
& =c|n|^{p}\|x\|^{p}+d|k|^{q}\|y\|^{q} \\
& \leq\left(|n|^{p}+|k|^{q}\right)\left(c\|x\|^{p}+d\|y\|^{q}\right) \\
& =\left(|n|^{p}+|k|^{q}\right) H(x, y)
\end{aligned}
$$

for every $x, y \in X \backslash\{0\}, k, n \in \mathbb{Z}$, and $k n \neq 0$. Hence,

$$
\begin{aligned}
\lim _{n \rightarrow \infty} & H\left(\mu_{n} x, \mu_{n}^{\prime} y\right) \\
\leq & \lim _{n \rightarrow \infty}\left(n^{p}+(n-1)^{q}\right) H(x, y) \\
& =0 \quad x, y \in X
\end{aligned}
$$

and there is $M>1$ such that

$$
\lambda\left(\mu_{n}\right)+\lambda\left(\mu_{n}^{\prime}\right)=n^{p}+(n-1)^{q}<\frac{1}{2} \quad n \in \mathbb{N}, n>M .
$$

So, it is easily seen that conditions (41) are fulfilled with

$$
\mathcal{U}:=\left\{\mu_{n} \in \text { Aut } X: n \in \mathbb{N}, n>M\right\}
$$

and therefore (by Corollary 6) every $f: X \rightarrow E$ satisfying (15), with $H$ given by (46) is additive.

Clearly, the above reasoning also works (after an easy modification) when the function $H:(X \backslash\{0\})^{2} \rightarrow \mathbb{R}_{+}$has the following a bit more involved form

$$
H(x, y)=c\|\eta(x)\|^{p}+d\|\chi(y)\|^{q} \quad x, y \in X \backslash\{0\},
$$

with some real $p<0, q<0, c \geq 0$, and $d \geq 0$ and additive injections $\eta, \chi: X \rightarrow E_{1}$ (or $\eta, \chi: X \rightarrow E$ ). So, we have the following corollary corresponding to the hyperstability results in $[16,21,24]$ (see also $[1,22,23,25])$.

Corollary 9. Let $H$ be given by (51) with some real $p<0$, $q<0, c \geq 0$, and $d \geq 0$ and some additive injections $\eta, \chi:$ $X \rightarrow E_{1}(\eta, \chi: X \rightarrow E$, resp.). Then every $f: X \rightarrow E$ satisfying (15) is additive.

We also get an analogous conclusion when $H$ is given by

$$
H(x, y)=c\|\eta(x)\|^{p}\|\chi(y)\|^{q} \quad x, y \in X \backslash\{0\},
$$

with some real $c>0$ and $p, q \in \mathbb{R}$ such that $p+q<0$ and some additive injections $\eta, \chi: X \rightarrow E_{1}$ (or $\eta, \chi: X \rightarrow E$ ), because

$$
\begin{aligned}
H(n x, k y) & =c\|\eta(n x)\|^{p}\|\chi(k y)\|^{q} \\
& =c|n|^{p}\|\eta(x)\|^{p}|k|^{q}\|\chi(y)\|^{q}
\end{aligned}
$$

for every $x, y \in X \backslash\{0\}, k, n \in \mathbb{Z}$, and $k n \neq 0$. So we have the following hyperstability result, as well (it generalizes to some extend the main outcome in [36]).

Corollary 10. Let $H$ be given by (52) with some $p, q \in \mathbb{R}$, $p+q<0, c \geq 0$, and some additive injections $\eta, \chi: X \rightarrow E_{1}$ $(\eta, \chi: X \rightarrow E$, resp.). Then every $f: X \rightarrow$ E satisfying (15) is additive.

It is easily seen that another example of the function $H$ satisfying (41) is given by

$$
H(x, y)=\left(c\|\eta(x)\|^{p}+d\|\chi(y)\|^{q}\right)^{r}, \quad x, y \in X \backslash\{0\},
$$

with some real $p>0, q>0, r<0, c \geq 0, d \geq 0, c+d>0$ and some additive injections $\eta, \chi: X \rightarrow E_{1}(\eta, \chi: X \rightarrow E$, resp.), because

$$
\begin{aligned}
H(n x, k y) & =\left(c|n|^{p}\|\eta(x)\|^{p}+d|k|^{q}\|\chi(y)\|^{q}\right)^{r} \\
& \leq\left(\min \left\{|n|^{p},|k|^{q}\right\}\right)^{r}\left(c\|\eta(x)\|^{p}+d\|\chi(y)\|^{q}\right)^{r} \\
& =\left(\min \left\{|n|^{p},|k|^{q}\right\}\right)^{r} H(x, y)
\end{aligned}
$$

for every $x, y \in X \backslash\{0\}, k, n \in \mathbb{Z}$, and $k n \neq 0$. So, we have yet the following.

Corollary 11. Let $H$ be given by (54) with some real $p>0$, $q>0, r<0, c \geq 0, d \geq 0, c+d>0$ and some additive injections $\eta, \chi: X \rightarrow E_{1}(\eta, \chi: X \rightarrow E$, resp.). Then every $f: X \rightarrow$ E satisfying (15) is additive.

We finish the paper with an example of corollary that generalizes some results in [37] and improves the estimations obtained there.

Corollary 12. Let $X$ be divisible by 2 and let $H$ be given by (51) with some real numbers $1<p<q, c \geq 0$, and $d \geq 0$ and some additive injections $\eta, \chi: X \rightarrow E_{1}(\eta, \chi: X \rightarrow E$, resp.). Then, for every $f: X \rightarrow E$ satisfying (15), there exists an additive mapping $T: X \rightarrow E$ such that

$$
\|f(x)-T(x)\| \leq \frac{c\|\eta(x)\|^{p}+2^{p-q} d\|\chi(y)\|^{q}}{2^{p}-2}, \quad x \in X .
$$


Proof. Let $f: X \rightarrow E$ satisfy (15) and $u_{0}: X \rightarrow X$ be given by

$$
u_{0}(x)=\frac{1}{2} x \quad x \in X
$$

Then $u_{0}=u_{0}^{\prime}$ and $\lambda\left(u_{0}\right) \leq 2^{-p}$. Consequently, by Theorem 5 with $\mathscr{U}=\left\{u_{0}\right\}$, there is a unique additive $T: X \rightarrow E$ such that

$$
\begin{aligned}
\|f(x)-T(x)\| & \leq \frac{c\left\|\eta\left(u_{0} x\right)\right\|^{p}+d\left\|\chi\left(u_{0}^{\prime} y\right)\right\|^{q}}{1-2 \lambda\left(u_{0}\right)} \\
& \leq \frac{2^{-p} c\|\eta(x)\|^{p}+2^{-q} d\|\chi(y)\|^{q}}{1-2^{1-p}} \\
& =\frac{c\|\eta(x)\|^{p}+2^{p-q} d\|\chi(y)\|^{q}}{2^{p}-2} \quad x \in X .
\end{aligned}
$$

\section{Conflict of Interests}

The author declares that there is no conflict of interests regarding the publication of this paper.

\section{References}

[1] N. Brillouët-Belluot, J. Brzdęk, and K. Ciepliñski, "On some recent developments in Ulam's type stability," Abstract and Applied Analysis, vol. 2012, Article ID 716936, 41 pages, 2012.

[2] S. Czerwik, Functional Equations and Inequalities in Several Variables, World Scientific, London, UK, 2002.

[3] V. A. Faĭziev, T. M. Rassias, and P. K. Sahoo, "The space of $(\psi, \gamma)$-additive mappings on semi-groups," Transactions of the American Mathematical Society, vol. 354, pp. 4455-4472, 2002.

[4] D. H. Hyers, G. Isac, and T. M. Rassias, Stability of Functional Equations in Several Variables, Birkhäuser, Boston, Mass, USA, 1998.

[5] S.-M. Jung, Hyers-Ulam-Rassias Stability of Functional Equations in Mathematical Analysis, Hadronic Press, Palm Harbor, Fla, USA, 2001.

[6] S.-M. Jung, Hyers-Ulam-Rassias Stability of Functional Equations in Nonlinear Analysis, vol. 48 of Springer Optimization and Its Applications, Springer, London, UK, 2011.

[7] Z. Moszner, "On the stability of functional equations," Aequationes Mathematicae, vol. 77, no. 1-2, pp. 33-88, 2009.

[8] Z. Moszner, "On stability of some functional equations and topology of their target spaces," Annales Universitatis Paedagogicae Cracoviensis Studia Mathematica, vol. 11, pp. 69-94, 2012.

[9] D. H. Hyers, "On the stability of the linear functional equation," Proceedings of the National Academy of Sciences of the USA, vol. 27, pp. 222-224, 1941.

[10] S. M. Ulam, A Collection of Mathematical Problems, Interscience Publishers, New York, NY, USA, 1960.

[11] S. M. Ulam, Problems in Modern Mathematics, John Wiley and Sons, New York, NY, USA, 1964.

[12] T. M. Rassias, "On the stability of the linear mapping in Banach spaces," Proceedings of the American Mathematical Society, vol. 72, pp. 297-300, 1978.
[13] T. M. Rassias, “Problem," Aequationes Mathematicae, vol. 39, p. 309, 1990.

[14] T. M. Rassias, “On a modified Hyers-Ulam sequence," Journal of Mathematical Analysis and Applications, vol. 158, no. 1, pp. 106113, 1991.

[15] T. Aoki, "On the stability of the linear transformation in Banach spaces," Journal of the Mathematical Society of Japan, vol. 2, pp. 64-66, 1950.

[16] J. Brzdęk, "Hyperstability of the Cauchy equation on restricted domains," Acta Mathematica Hungarica, vol. 141, pp. 58-67, 2013.

[17] Z. Gajda, "On stability of additive mappings," International Journal of Mathematics and Mathematical Sciences, vol. 14, pp. 431434, 1991.

[18] T. M. Rassias and P. Semrl, "On the behavior of mappings which do not satisfy Hyers-Ulam stability," Proceedings of the American Mathematical Society, vol. 114, pp. 989-993, 1992.

[19] T. M. Rassias and P. Semrl, "On the Hyers-Ulam Stability of Linear Mappings," Journal of Mathematical Analysis and Applications, vol. 173, no. 2, pp. 325-338, 1993.

[20] J. Brzdęk, "A note on stability of additive mappings," in Stability of Mappings of Hyers-Ulam Type, T. M. Rassias and J. Tabor, Eds., pp. 19-22, Hadronic Press, Palm Harbour, Fla USA, 1994.

[21] J. Brzdęk, "Remarks on hyperstability of the Cauchy functional equation," Aequationes Mathematicae, vol. 86, pp. 255-267, 2013.

[22] J. Brzdęk and K. Ciepliñski, "Hyperstability and superstability," Abstract and Applied Analysis, vol. 2013, Article ID 401756, 13 pages, 2013.

[23] A. Bahyrycz and M. Piszczek, "Hyperstability of the Jensen functional equation," Acta Mathematica Hungarica, vol. 142, pp. 353-365, 2014.

[24] M. Piszczek, "Remark on hyperstability of the general linear equation," Aequationes Mathematicae.

[25] M. Piszczek and J. Szczawińska, "Hyperstability of the Drygas functional equation," Journal of Function Spaces and Applications, vol. 2013, Article ID 912718, 4 pages, 2013.

[26] J. Brzdęk and K. Ciepliñski, "A fixed point approach to the stability of functional equations in non-Archimedean metric spaces," Nonlinear Analysis: Theory, Methods and Applications, vol. 74, no. 18, pp. 6861-6867, 2011.

[27] J. Brzdęk, J. Chudziak, and Z. Páles, "A fixed point approach to stability of functional equations," Nonlinear Analysis: Theory, Methods and Applications, vol. 74, no. 17, pp. 6728-6732, 2011.

[28] L. Cădariu, L. Găvruţa, and P. G. Găvruţa, "Fixed points and generalized Hyers-Ulam stability," Abstract and Applied Analysis, vol. 2012, Article ID 712743, 10 pages, 2012.

[29] K. Ciepliñski, "Applications of fixed point theorems to the Hyers-Ulam stability of functional equations: a survey," Annals of Functional Analysis, vol. 3, pp. 151-164, 2012.

[30] C. Borelli Forti, "Solutions of a nonhomogeneous Cauchy equation," Radovi Matematicki, vol. 5, pp. 213-222, 1989.

[31] B. Ebanks, "Generalized Cauchy difference functional equations," Aequationes Mathematicae, vol. 70, no. 1-2, pp. 154-176, 2005.

[32] B. Ebanks, "Generalized Cauchy difference equations. II," Proceedings of the American Mathematical Society, vol. 136, no. 11, pp. 3911-3919, 2008.

[33] B. Ebanks, P. Kannappan, and P. K. Sahoo, "Cauchy differences that depend on the product of arguments," Glasnik Matematicki, vol. 27 (47), pp. 251-261, 1992. 
[34] I. Fenyö and G. L. Forti, "On the inhomogeneous Cauchy functional equation," Stochastica, vol. 5, pp. 71-77, 1981.

[35] A. Járai, G. Maksa, and Z. Páles, "On Cauchy-differences that are also quasisums," Publicationes Mathematicae, vol. 65, no. 34, pp. 381-398, 2004.

[36] J. Brzdęk, "A hyperstability result for the Cauchy equation," Bulletin of the Australian Mathematical Society, vol. 89, no. 1, 2014.

[37] G. Isac and Th. M. Rassias, "Functional inequalities for approximately additive mappings," in Stability of Mappings of HyersUlam Type, T. M. Rassias and J. Tabor, Eds., pp. 117-125, Hadronic Press, Palm Harbour, Fla, USA, 1994. 


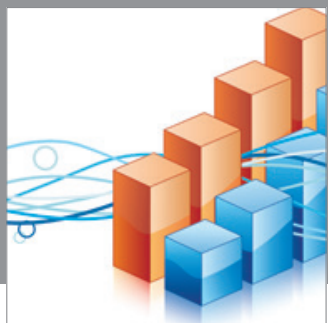

Advances in

Operations Research

mansans

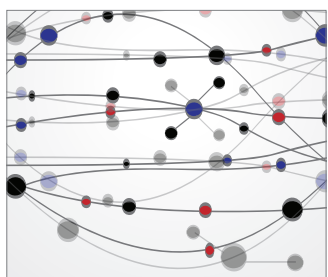

The Scientific World Journal
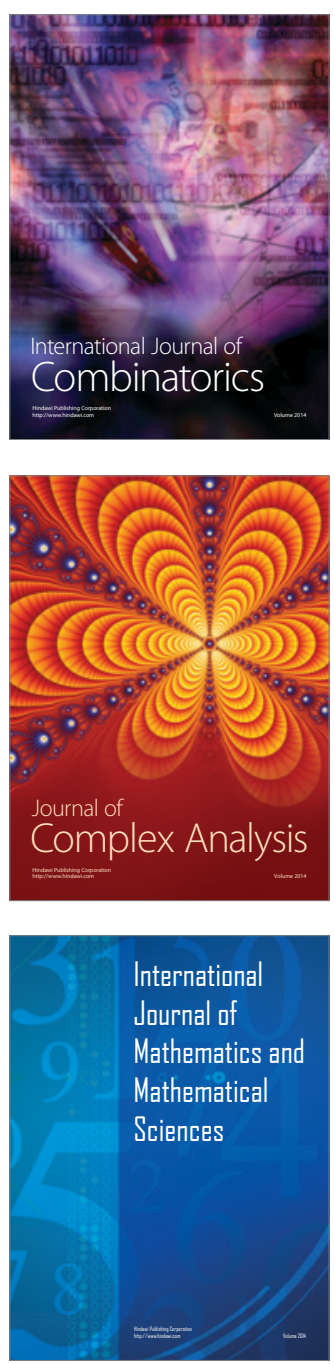
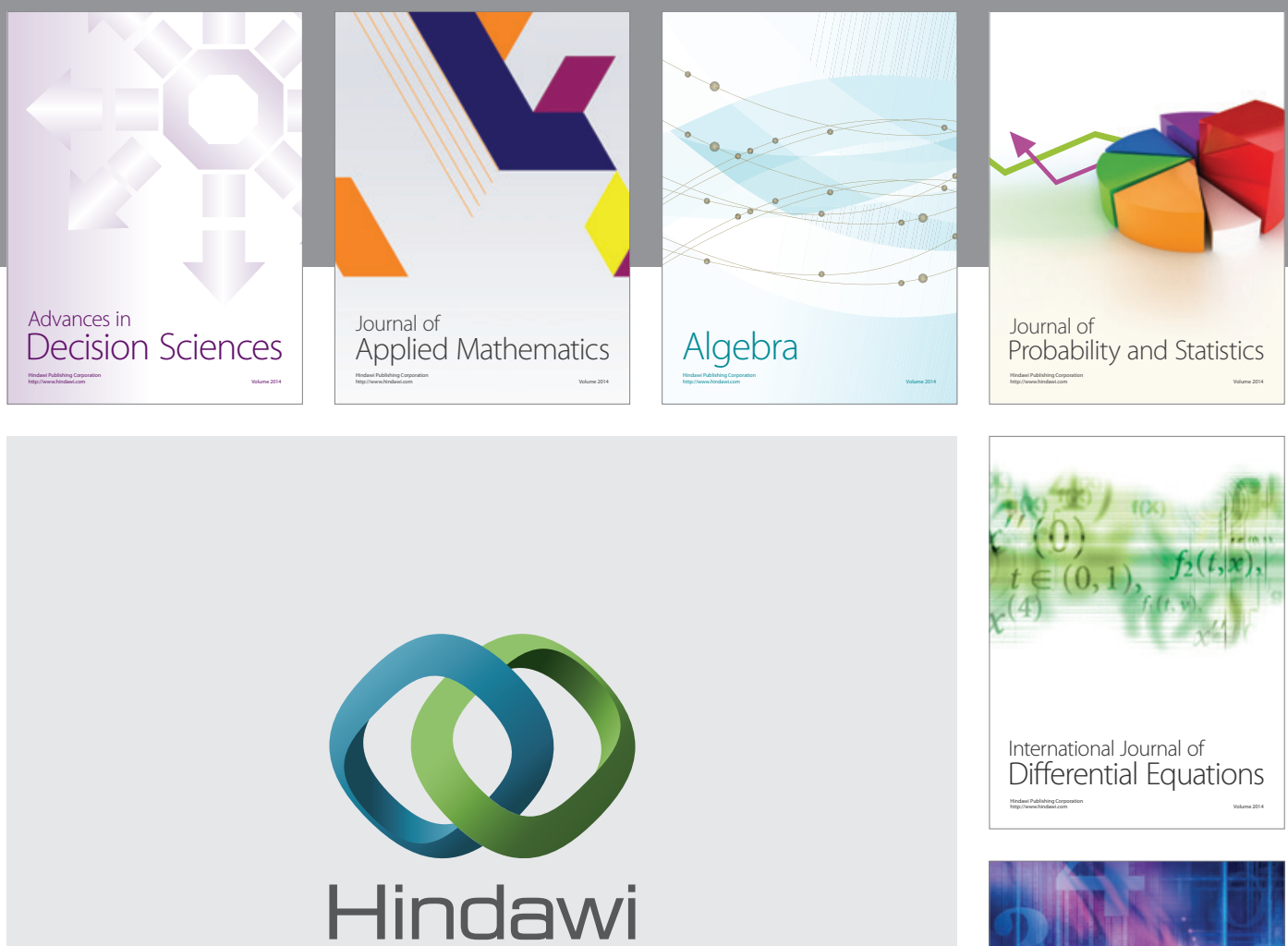

Submit your manuscripts at http://www.hindawi.com
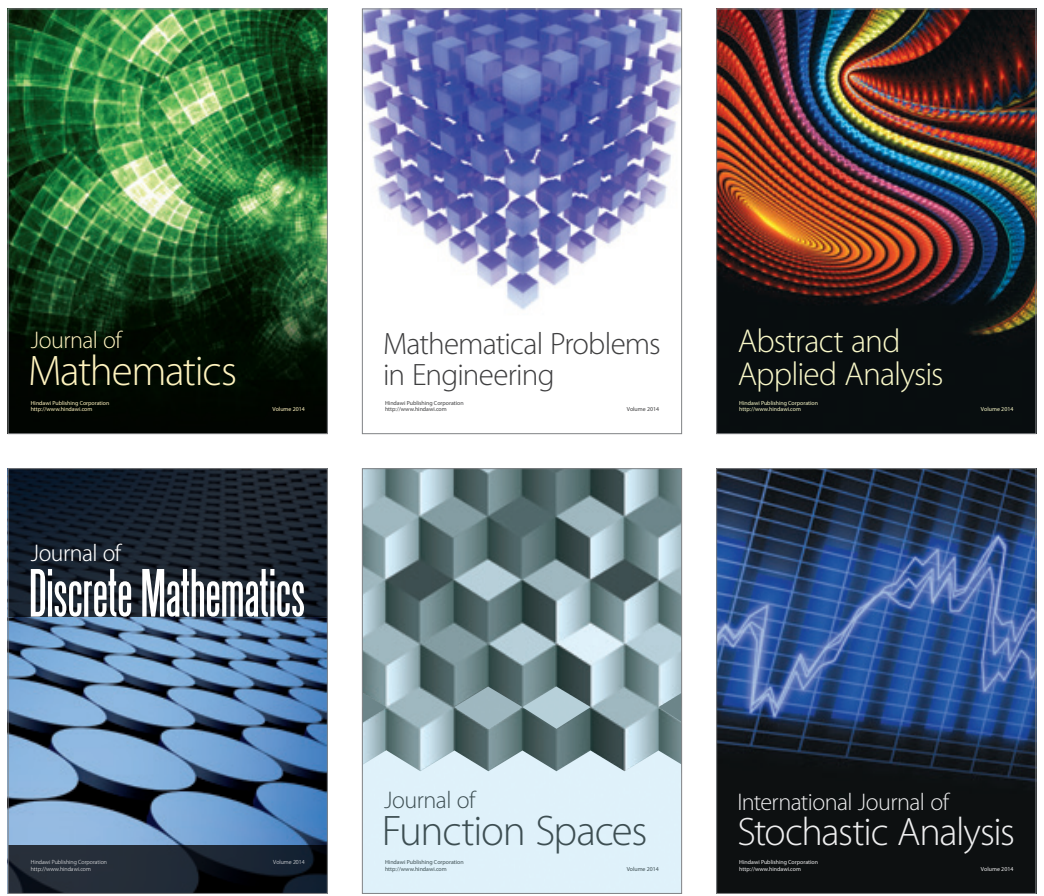

Journal of

Function Spaces

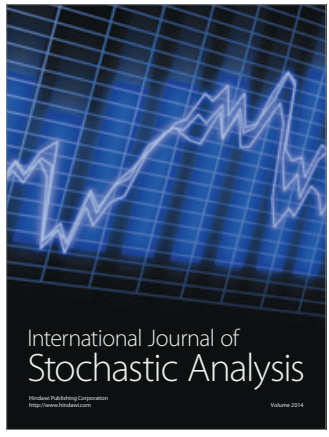

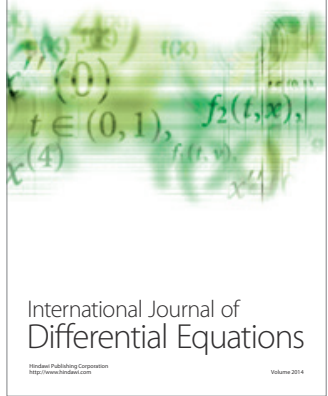
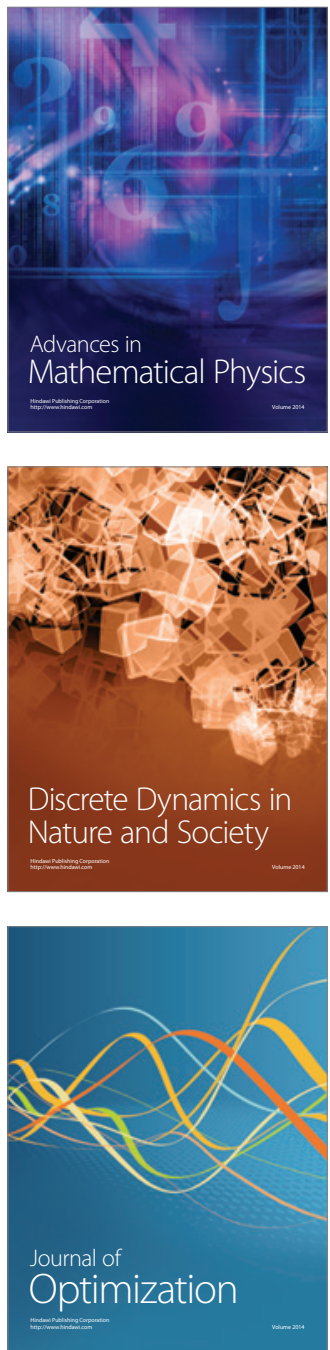